ARTícUlo

\title{
Los principios de la complejidad y su aporte al proceso de Enseñanza*
}

Alex Estrada García ${ }^{a}$

\section{Resumen}

El objetivo de este ensayo es plantear una ecología de saberes entre los principios de la complejidad y los paradigmas considerados como tradicionales. En primera instancia se ofrece una perspectiva de lo que se entiende por complejidad, seguida de un breve abordaje sobre el proceso de Enseñanza, y por último se realiza una sistematización de conceptos entre los principios de la complejidad y el proceso de Enseñanza. Como método de investigación se utilizó la hermenéutica. En conclusión, el documento integra un diálogo epistemológico que guía a repensar la Educación, específicamente el proceso de Enseñanza, desde una perspectiva compleja y transdisciplinar.

Palabras clave: Educación Superior. Pensamiento Complejo. Proceso de Enseñanza. Principios del Pensamiento Complejo.

\section{Introducción}

Hoy en día, la sociedad ha incrementado la complejidad del mundo. Comprenderlo, analizarlo y vivirlo implica construir, desde la alteridad, una nueva forma de pensar lo pensado (GONFIANTINI, 2015). Es evidente la necesidad de una Educación que permita reaprender conocimientos apegados a la realidad en la que se vive, que logre comprender este mundo cambiante y complejo, porque a partir de la complejidad de los sujetos u objetos se puede empezar a reorganizar la Educación desde un enfoque sistémico, transdisciplinar y crítico, con la finalidad de poner en práctica una Enseñanza y aprendizaje que no mutile el conocimiento y enseñe a convivir en un ambiente de armonía.

En el campo educativo se han realizado múltiples estudios; se toma como ejemplo los siguientes: Bohórquez Arenas y D’amore (2018), Cedeño Álvarez, Guarnizo

\footnotetext{
* Este artículo es el resultado de la construcción teórica de una investigación doctoral titulada "la Educación transdisciplinar como una vía para generar conocimientos dentro de la formación de pedagogos".

a Universidad Nacional de Chimborazo, Riobamba, Ecuador.
} 
Delgao y Pacheco Proaño (2018), Himmel (2011), Borrero Springer y Gamboa Graus (2017) y Tejedor Tejedor (2018), analizan el proceso de Enseñanza, corroborando que existe una insuficiencia del sistema educativo al momento de realizar el proceso de Enseñanza y aprendizaje, en gran parte debido al dominio del paradigma cartesiano positivista y reductor dentro de la Educación, que no permite la comprensión de la incertidumbre, ni tampoco el reconocimiento a lo irreductible; es decir, este paradigma no permite ingresar a la complejidad existente en el sistema educativo. Para poder comprender esta complejidad, Morin (1994) propone un método distinto a los utilizados por la ciencia clásica, universal, sistémico, ecologizado y, sobre todo, que comprenda la realidad.

Además, Edgar Morin discierne que la base de la crisis que está atravesando la humanidad es una crisis cognitiva, por lo que afirma que "[...] nuestro modo de conocimiento no ha desarrollado suficientemente la aptitud para contextualizar la información e integrarla en un conjunto que le dé sentido" (MORIN, 2011, p. 142). Se considera crisis cognitiva, puesto que no se tiene la capacidad de abordar un determinado problema desde diferentes perspectivas. Esto se debería a la sobreabundancia de información, a la fragmentación, a las disciplinas que no se comunican, a un "[...] predominio de la hiperespecialización, que rompe el tejido complejo de lo real, y con un predominio de lo cuantificable que solo busca asegurar en el sistema de Educación lo que algunos llaman cientificidad a través de la medición [...]" (YANES, 2015, p. 87).

En el contexto nacional, como consecuencia del agotamiento paradigmático, las instituciones de Educación Superior del Ecuador a partir del año 2015 se vieron en la necesidad de generar un cambio en los modelos educativos. Este cambio exigía replantear la forma de cómo se estaba educando y las metodologías que se utilizaban para el proceso de Enseñanza, con la finalidad de brindar una calidad educativa y contribuir de forma integral en la formación de profesionales.

Para hacer posible este cambio, se construyeron nuevas mallas curriculares de las carreras ofertadas por las universidades; estas mallas fueron desarrolladas para dar respuesta a la exigencia de la sociedad actual, por ende, la forma de aprender y enseñar tuvieron un cambio significativo: los docentes debían abandonar el paradigma cartesiano positivista, con el que se venía trabajando durante muchos años, basado en la disciplinariedad, llevándonos a la especialización en ciertas áreas de conocimiento y permitiendo la fragmentación del mismo.

Por otro lado, por parte de los docentes existe un descuido en cuanto a la búsqueda metodológica que garantice una Enseñanza de calidad, acorde a lo requerido por la 
sociedad. Es evidente la creciente necesidad de reorientar el horizonte educativo; como muestra de esta necesidad, la Organización de las Naciones Unidas para la Educación, la Ciencia y la Cultura (UNESCO, 2015) publica un documento llamado: Replantear la Educación, ¿hacia un bien común?, en el que se considera que la Educación debe dirigirse hacia un desarrollo de competencias, tomando en cuenta la complejidad del mundo actual. El planteamiento de la Unesco es una de las muchas manifestaciones en cuanto a las emergencias que está atravesando la Educación; por ello, es evidente la necesidad de replantear la forma de educar para dar respuesta a los constantes cambios socioculturales, epistemológicos, ambientales e históricos.

Para iniciar la construcción de este camino regenerador de procesos dentro de la Educación, se considera oportuno ayudarse de paradigmas como el crítico, complejo, sistémico, que conduzcan hacia la creación de un pensamiento transdisciplinar, dinámico, abierto a nuevos desafíos, que trascienda el paradigma de la simplificación y disyunción.

Se tiene claro que el cambio de paradigma crea la necesidad de una transformación metodológica dentro de la Educación, empezando desde la redefinición y planificación de las diferentes asignaturas, como a la innovación de estrategias metodológicas por parte de los docentes. Un ejemplo claro, es lo exigido por el Consejo de Educación Superior (2015) a todas las universidades del sistema nacional de Educación del Ecuador: crear un currículo universitario desde un enfoque sistémico, y con base a esto, reorganizar las mallas curriculares de todas las carreras, con la finalidad de crear conexiones entre la universidad y la sociedad, para construir aprendizajes significativos que ayuden a resolver los problemas de la vida real.

\section{Desarrollo}

\section{1 ¿Qué es el pensamiento complejo?}

Morin (1995) propone la idea de complejidad como una articulación de los fenómenos del mundo. Este modo de pensar implica un "alto grado de desorden debido a todo lo que abarcaría, de allí la vocación histórica del conocimiento científico por buscar cierto orden" (VANOLI, 2017, p. 4). El método científico siempre ha pretendido reducir dicha incertidumbre y ambigüedad, mientras que el paradigma del pensamiento complejo invita a conocer la imposibilidad de simplificar, por lo que direcciona a buscar el fundamento central de los problemas. 
En este sentido, al pensamiento complejo se considera como un paradigma epistemológico que defiende una estrategia fundamental, llamada reforma del pensamiento, el cual "concibe a la realidad como un sistema en permanente cambio, desarrollo y emergencia; reconoce al todo como la suma de sus partes y a la especificidad de las partes respecto al todo" (GÓMEZ; HERNÁNDEZ; RAMOS, 2016, p. 474). La reforma del pensamiento propuesta por Morin aspira a la superación de un pensamiento simplificador, y al mismo tiempo, de un "principio del pensamiento que opera a partir de una lógica disyuntora-reductora, de una explicación racional del mundo que se instauró con el pensamiento científicoclásico" (OSORIO GARCÍA, 2012, p. 273).

Siguiendo la historia, a finales del siglo XX e inicios del siglo XXI empezaba a ser cuestionado el pensamiento cartesiano positivista, pero a la par, se proporcionaba el saber necesario para dar origen a una transformación paradigmática del conocimiento. Hoy en día, esta transformación se pretende alcanzar mediante la articulación del conocimiento obtenido desde la diversidad de corrientes, teorías, enfoques y paradigmas existentes, con la finalidad de encontrar una comprensión compleja de lo real.

En torno al paradigma de la complejidad, Gómez, Hernández y Ramos (2016, p. 473), discierne que "la complejidad en Morin no trata de los asuntos propios de las así llamadas ciencias de la complejidad, sino, de un nuevo horizonte epistemológico para organizar el pensamiento". Esta organización del pensamiento permitirá el acceso a la creación de conocimientos que posibiliten la emergencia de una nueva manera de ser, pensar, actuar y convivir, con la finalidad de una transformación en la sociedad.

La epistemología compleja en la construcción de conocimiento gira alrededor del problema de la verdad, pasando de perspectiva en perspectiva, de verdades parciales, intentando efectuar una rearticulación del saber, del conocimiento inseparable de una reflexión fundamental. Para mantener abierta la problemática de la verdad, considera cualquier conocimiento, ya sea epistémico o extraepistémico que cree verdadero, cualquier presunción de conocimiento, incluyendo el error, la ilusión, el desconocimiento (TORREALBA; PÉREZ; CASTILLO, 2018, p. 140).

En concordancia, se considera que la complejidad interpreta y relaciona al mundo como un tejido conformado de múltiples uniones que se enlazan entre sí para formar relaciones sistematizadas. Por ejemplo, dentro de esta 
forma de pensar podemos abordar una diversidad de conocimientos que se interconectan formando una red compleja, así tenemos, el estudio de la vida, los problemas sociales, el futuro de la humanidad, las relaciones existentes entre los seres vivos, etc.

Además, al pensamiento complejo se puede entender como lo que permite la integración de lo humano, considerándolo como elemento constitutivo y constituyente de la complejidad. Por ende, este paradigma no es un discurso terminado, acabado, cerrado, sino una orientación hacia aquello que tenemos que volver la mirada si queremos asegurar la supervivencia de la humanidad en la era planetaria (OSORIO GARCÍA, 2012).

\subsection{El proceso de Enseñanza en las Instituciones de Educación Superior}

Según Zabalza (2011, p. 191) el proceso de Enseñanza "es la reconsideración constante, mediante los cuales los estudiantes llegan al aprendizaje". Dentro del proceso de Enseñanza y aprendizaje existe una relación docente-estudiante, en la que al docente se lo considera como el guía del proceso de Enseñanza y encargado de crear un ámbito de confianza dentro del aula y de crear metodologías que faciliten la Enseñanza y despierten el interés por aprender.

Una metodología que se está trabajando dentro del sistema educativo ecuatoriano es la interdisciplinaria, que permite comprender los problemas sociales, culturales, ambientales, desde una forma sistémica, integrando varios conocimientos, formas de conocer, hacer, vivir, y ser. Con esta metodología se construye una experiencia socio-histórica, entre estudiante y sociedad, cuya interacción dará como resultado cambios que le permiten al estudiante adaptarse a la realidad, transformarla y crecer con una forma de pensar integral.

En esta encrucijada no se debe descuidar los avances tecnológicos, pues es vital para el proceso de Enseñanza; aquí, el rol del docente es orientar a que el estudiante haga uso adecuado de los recursos tecnológicos para mejorar su aprendizaje En palabras de la Unesco (2004), en la Educación, los objetivos estratégicos se direccionan a mejorar la calidad, por medio de la innovación de contenidos y métodos, de la promoción de la experimentación, la difusión y el uso compartido de las Tecnologías de la Información y Comunicación, de la realización de buenas prácticas, de la formación de redes de aprendizaje, y del estímulo de un diálogo fluido sobre las metodologías a seguir. En este sentido, también podemos mencionar que: 
La Educación está marcada por las tecnologías de la información y la comunicación y sobre todo por el desarrollo de Internet, que permanece a la cabeza de la revolución tecnológica produciendo importantes cambios en la formación, en las formas de comunicación, en el acceso y uso de la información (GUZMÁN, 2011, p. 56).

En concordancia con la cita, se discierne que con los avances tecnológicos, el campo educativo ha cambiado de forma radical, por lo que el docente deja de ser el protagonista principal del proceso de Enseñanza y aprendizaje cuya característica era exponer sus clases de forma magistral; en esta época se da paso a que el estudiante tome el rol principal y genere sus propios conocimientos, guiado por el docente en un entorno interactivo del proceso de Enseñanza y aprendizaje.

Con el acceso a la tecnología y a la diversidad de información, se crea un puente entre la Educación y la sociedad del conocimiento, permitiendo acercarse a las diferentes realidades globales, a numerosos paradigmas, lo que permite comprender al mundo desde una forma global y sistémica; pero hay que tener en cuenta que "uno de los desafíos más difíciles será el de modificar nuestro pensamiento de manera que enfrente la complejidad creciente, la rapidez de los cambios y lo imprevisible que caracterizan nuestro mundo" (MORIN, 1999, p. 32)

Lo propuesto por Morin invita a considerar la organización del conocimiento, a reaprender nuevos métodos que sirvan de ayuda para enfrentar la incertidumbre creciente en el sistema educativo y comprender que el proceso de Enseñanza y aprendizaje está llamado a ser sistémico, crítico y transdisciplinar, donde se posibilite al estudiante la apropiación activa y dinámica del conocimiento, sistematizando la ciencia, tecnología y sociedad, sin descuidar aspectos claves como la ética y los valores.

En la misma línea de análisis, Peraza Zamora et al. (2017) manifiestan que el objetivo del proceso de Enseñanza y aprendizaje es fortalecer el:

[...] crecimiento humano para contribuir a la formación y desarrollo de una personalidad auto determinada, pero para que este proceso se lleve a cabo con calidad y cumpla con estos objetivos es preciso exista una estrecha relación entre los componentes didácticos que lo forman; entre los que se encuentran los medios de Enseñanza (PERAZA ZAMORA et al., 2017, p. 5). 
Además, se puede considerar al proceso de Enseñanza y aprendizaje como la capacidad cognoscitiva de los estudiantes que, bajo la orientación del docente, tiene como finalidad alcanzar el dominio de conocimientos científicos, el desarrollo de habilidades, y la articulación de los saberes propios de los estudiantes con las diferentes perspectivas científicas del mundo. Dentro de este proceso existe una diversidad de problemáticas que no han podido ser resueltas, y que inducen al proceso de Enseñanza y aprendizaje a caer en la monotonía, donde el estudiante cada vez se muestra descontento con el sistema educativo.

Un docente puede enseñar bien y el alumno no aprender; por otra parte, un alumno puede aprender, a pesar de que existan falencias por parte del docente; en este sentido, Bisquerra Alzina (2012, p. 37) considera que "el aprendizaje es lo que justifica el sistema educativo; no la Enseñanza". Claramente lo que le importa al sistema educativo es un resultado cuantitativo, no el desarrollo del proceso de Enseñanza y aprendizaje.

Apegando el análisis al contexto de la Educación Superior del Ecuador, el proceso de Enseñanza y aprendizaje se basa en dos criterios de calidad: entornos de los aprendizajes y logros de los aprendizajes. Existen varios documentos que los sustentan, entre otros: Constitución de la República del Ecuador (2008), Ley Orgánica de Educación Superior (2010), Reglamento de la Ley Orgánica de Educación Superior (2012), Plan Nacional del Buen Vivir, Plan Nacional de Ciencia, Tecnología, Innovación y Saberes Ancestrales.

Estos criterios han sido fundamentales en el desarrollo de la Educación Superior del Ecuador, influyendo directamente en el proceso de Enseñanza; un ejemplo es lo realizado por la Universidad Nacional de Chimborazo en la Facultad de Ciencias de la Educación, Humanas y Tecnología, que promovió un cambio en el modelo educativo. Para aplicar este cambio dentro del proceso de Enseñanza y aprendizaje, se modificó las mallas curriculares de todas las carreras, implementando un programa de estudio de asignaturas con criterio de competencias sistémicas. Se debe tener en cuenta que para lograr un buen funcionamiento del modelo de Enseñanza se requiere que este sea integrador, sistémico, transdisciplinario (MORIN, 1994).

\subsection{Principios del pensamiento complejo y su relación con el proceso de Enseñanza}

Edgar Morin, considerado como un pensador planetario, plantea los siete principios del pensamiento complejo, con el propósito de reformar el pensamiento y lograr romper el paradigma tradicionalista; estos principios guían a la construcción de 
un conocimiento sistémico, que posibilitan interacciones, retroacciones y afrontar las incertidumbres; partiendo de un pensamiento de lo complejo, el cual va a reformar la manera de pensar, por medio de la integración de saberes dispersos, que totalicen la información y los conocimientos, articulando lo que está fragmentado, a través de una reorganización teórica (ESTRADA GARCIA, 2018a).

Ante la idea planteada, se añade lo expuesto por Morin (1994, p. 140), que aclara que la "complejidad no es un fundamento, es el principio regulador que no pierde nunca de vista la realidad del tejido fenoménico en la cual estamos y que constituye nuestro mundo". Por ello, brinda un método para comprender las coyunturas existentes en una relación de sistema complejo entre el ser humano, la naturaleza, la sociedad y la cultura.

Para ejemplificar lo expuesto, se plantea lo siguiente: la naturaleza en sí, es un ejemplo de complejidad, pero también es un ejemplo de nobleza, grandeza y poder, que va más allá de los sectores económicos, políticos, sociales o tecnológicos. La fuerza de un tornado, un terremoto o tsunami y la paz, la tranquilidad y la serenidad son dadas por la naturaleza y no existe nada que pueda reemplazar o imitar estos hechos.

Dentro del campo educativo, los principios del pensamiento complejo servirán para crear ambientes de aprendizajes apegados a la realidad del estudiante. También, este pensamiento permitirá al docente crear estrategias para el diseño de metodologías asertivas para la Enseñanza, tomando en cuenta la aparición de nuevos campos como es la neuroeducación, a la que Serghie (2015); Maasen y Lieven (2016) consideran como un campo transdisciplinar, y la Era digital.

Con los principios del pensamiento complejo se pretende establecer una complementariedad entre diferentes teorías, paradigmas y enfoques; como resultado se podrá crear un ambiente de aprendizaje donde se permita al estudiante la apropiación del conocimiento, adquisición de habilidades y valores, obteniendo un aprendizaje duradero, que ayudará a desenvolverse de una forma asertiva en su interacción con la sociedad.

\subsubsection{Principio sistémico u organizativo}

En relación a este principio, Morin (1994) destaca lo siguiente “[...] la organización de un todo produce cualidades o propiedades nuevas en relación con las partes consideradas de forma aislada" (p. 69). Este principio permite articular el conocimiento de las partes con el del todo, y su objetivo radica en combatir la idea reduccionista, de que el todo es tan solo la suma de las partes. 
Desde esta perspectiva, la Enseñanza en los entornos educativos no es solo la responsabilidad de los docentes, sino de todo el sistema educativo. Para que la Enseñanza sea de calidad, el sistema educativo debe proporcionar los instrumentos necesarios, y todos sus componentes están llamados a funcionar de manera articulada, guiando a un correcto funcionamiento de todo el sistema educativo, sin dar mayor valor a ciertos componentes, sino de manera equitativa. Todo esto, con la finalidad de construir un conocimiento duradero que posibilite repensar los problemas socioeducativos.

Con el objetivo de mejorar el funcionamiento del sistema educativo, especialmente el proceso de Enseñanza, es necesario reconstruir el currículo educativo, pues se encuentra alejado de la realidad, no contextualiza las necesidades de la sociedad y tampoco muestra una conexión entre sociedad-Educación-currículo. Ante esto, es oportuno trabajar en el currículo sistémico que se fundamente en una concepción global del aprendizaje, tratando de desarrollar tanto los conceptos, actitudes y metodología.

\subsubsection{Principio hologramático}

Inspirado "en el holograma, en el que cada punto contiene la casi totalidad de la información del objeto que representa" (MORIN, 2007, p. 99), este principio se considera como el punto medio entre el holismo y el reduccionismo; el holismo permite estudiar al objeto desde la parte metodológica y epistemológica, considerándole como una totalidad organizada sistémica, mas no como la suma de las partes; mientras que el reduccionismo se basa en el estudio del objeto como uno solo, aislando e ignorando ciertos componentes que lo conforman. Para lograr comprender la realidad, Morin propone estudiar con una visión holográmica, en donde las partes son de suma importancia como el todo. Además, Morin (1999, p. 6) afirma que:

Hay presencia del todo al interior de las partes [...] como cada punto singular de un holograma contiene la totalidad de la información de lo que representa, cada célula singular, cada individuo singular contiene de manera holográmica el todo del cual hace parte y que al mismo tiempo hace parte de él.

Esta perspectiva del objeto de estudio no fragmenta, permite llevar a cabo una comunicación que se puede percibir como una doble vía, en donde el todo se complementa con la información de las partes. En el campo de la Educación, este principio permite realizar una interacción entre las partes que componen el 
proceso de Enseñanza y aprendizaje tradicional, y buscar una salida valida hacia una nueva forma de pensar y organizar dicho proceso.

Sin embargo, esta interpretación holográmica requiere estimular un nuevo estilo de concebir el mundo, por lo que para construir este pensamiento es necesario no perder de vista las diferentes escalas de análisis de los fenómenos educativos, con la finalidad de interactuar con estos fenómenos y percibir las múltiples conexiones que se realizan entre estos, dando lugar a la formación de sistemas complejos, en donde se encuentran inmersos bucles retroactivos que pueden proporcionar una retroalimentación para solucionar algunas falencias encontradas.

\subsubsection{Principio del bucle retroactivo o retroalimentación}

Morin (1994, p. 99) destaca que "[...] la causa actúa sobre el efecto y el efecto sobre la causa, como un sistema de calefacción en el que el termostato regula el trabajo de la caldera [...]", en otras palabras, este principio facilita ver como la causa actúa sobre el efecto; rompiendo la idea de la causalidad lineal, permitiendo la expansión de la creación del conocimiento y la sistematización de los métodos.

En el campo educativo, este principio guía a la observación de la causalidad circular retroactiva del proceso de regulación de la dinámica estructural del sistema educativo, en donde los componentes educativos estarán en la capacidad de auto-organizarse, involucrando diferentes aspectos que contribuirían a la transformación educativa $y$, por ende, a la formación integral de los actores sociales inmersos en el proceso de Enseñanza y aprendizaje.

También, se añade lo expresado por Morin (2007, p. 99), en cuanto considera que la realimentación es el conocimiento de los procesos auto-reguladores que rompe con la causalidad lineal: la causa actúa sobre el efecto y el efecto sobre la causa, es una causalidad circular retroactiva. Este principio es comparable a la homeostasis que se pudiera producir en un organismo vivo, debido a que existe un proceso de autorregulación donde se llega a un punto cero de equilibrio o hay una exageración de ciertos niveles del mismo.

\subsubsection{Principio del bucle recursivo}

En palabras de Morin (2007, p. 28) la recursividad es uno de "los modos de pensar que [...] permitirían concebir que una misma cosa pueda ser causada y causante, ayudada y ayudantes, mediata e inmediata". En la realidad, esto se convierte en 
una constante de causa efecto, en donde se va multiplicando y se alimentan de la producción del nivel anterior; se puede decir que la causa actúa directamente sobre el efecto y viceversa.

Este principio, en la Educación, guía a una autoproducción y auto-organización de todos los componentes que influyen en los cambios paradigmáticos de la Educación, superando una noción de regulación, en donde los productos y efectos son los responsables de las causas producidas. También, orienta a la sociedad a que forme parte del sistema educativo, colaborando en la mejora de su calidad; y en conjunto reorganizando el proceso de Enseñanza y aprendizaje, de tal manera que se abandonen los métodos de la simplicidad y del reduccionismo, dando paso a una Educación reflexiva con un currículo sistémico, que guíe a la creación de competencias transdisciplinares en los futuros profesionales.

\subsubsection{Principio de autonomía/dependencia}

El ser humano pretende ser independiente sin perder la conexión con la sociedad, cultura y la naturaleza; por ello Morin también lo denomina principio de auto-ecoorganización. Morin (1994, p. 100) expone "[...] vivir de muerte, morir de vida [...]", según lo cual los seres vivientes se regeneran a partir de la muerte de sus células para darle origen a otras nuevas, y así mantener el equilibrio biológico; este principio pone en evidencia que los seres vivos tenemos la capacidad de poder desarrollarnos con autonomía.

Al respecto, se considera que la sociedad del conocimiento debe pensar una metodología que integre saberes científicos y no científicos (artísticos, ancestrales, religiosos, etc.) que ayude a crear proyectos que den solución a las problemáticas de la actualidad; una de las más abordadas en los últimos años es el cambio climático y la sobreexplotación de los recursos naturales, problemas que parecen ser cada día más grandes y no tener una solución efectiva. Ante estos problemas existe la necesidad de cambiar la forma de pensar de la sociedad, y esto se logra a través del cambio en la Educación.

Es evidente que la sociedad no está siendo educada desde la parte humanista, con una conciencia que invite a reflexionar de que todos somos seres vivos y debemos aprender a convivir; de ahí nace la urgencia de plantear un diálogo de saberes sobre la Educación que nos hace falta para el siglo XXI, la que dé respuestas a la diversidad de interrogantes que la sociedad se plantea, con base en diversidad, sostenibilidad, economía y calidad de vida. 


\subsubsection{Principio dialógico}

Este principio se opone a la disyunción y abstracción, porque establecen leyes generales, aíslan los objetos de estudio y excluyen las relaciones del contexto con el objeto estudiado. Para poder concebir lo dialógico y lo complejo debemos entender que son "[...] dos principios o nociones que deberían excluirse entre sí pero que son indisociables en una misma realidad" (MORIN, 2007, p. 100). En este caso por medio de lo dialógico, dialogan las partes con el todo y "[...] permite asumir racionalmente la inseparabilidad de nociones contradictorias para concebir un mismo fenómeno complejo [...]" (MORIN, 1994, p. 101).

Lo mencionado invita a concebir una dialógica orden/desorden/organización que se encuentra en constante interacción, a través de múltiples retroacciones en el mundo físico, biológico y humano. Mediante este principio se puede conocer que la dialógica permite asumir racionalmente la inseparabilidad de unas nociones contradictorias para el estudio de un mismo fenómeno complejo.

Desde esta perspectiva, la transformación educativa debería enfocarse en objetivos que estén al alcance del proceso de Enseñanza y aprendizaje, y capacitar a los docentes para que desarrollen una metodología sistémica, que posibilite integrar las fragmentaciones, con la finalidad de estudiarlas como un sistema, teniendo en cuenta los cuatro pilares fundamentales para la Educación expuestos por la Unesco (aprender a conocer, aprender a ser, aprender hacer y aprender a vivir juntos). Haciendo referencia a lo antes expuesto, Morin (1999, p. 45) manifiesta que:

[...] hay que substituir la visión de un universo que obedece a un orden impecable por una visión donde el universo sea el juego y lo que está en juego de una dialógica (relación antagónica, competente y complementaria) entre el orden, el desorden y la organización. La Tierra, probablemente en sus inicios, un montón de desperdicios cósmicos procedentes de una explosión solar, se organizó así misma en una dialógica entre orden - desorden - organización.

Tomando como ejemplo la cita expuesta anteriormente, se relaciona el proceso de cambios que se ha venido dando en la Educación, se ve afectada por los modelos caducos de Enseñanza, en donde existe una divergencia entre la metodología y los contenidos a enseñar, siendo estos últimos enseñados por parte del docente de una forma fragmentada, llevando al estudiante a comprender solo ciertas áreas disciplinarias, las que no son suficientes para ayudar a la formación de profesionales en el siglo XXI. 
En la actualidad, es necesario crear en el estudiante emociones, así lo afirma Mora (2019), pues esto facilita el aprendizaje, y permite fortalecer diferentes características: aptitudes, capacidades, habilidades, motivación, personalidad, valores, etc. Por lo que es necesario reestructurar los modelos educativos, con el objetivo de establecer un diálogo de saberes transdisciplinares, para que el sistema educativo sea asertivo en el proceso de Enseñanza y aprendizaje, desarrollando en el estudiante conocimientos que le permitan solucionar la diversidad de problemas relacionados con su entorno.

\subsubsection{Principio del que conoce en todo conocimiento}

Este principio refiere a que todo conocimiento es una reconstrucción/traducción que lleva a cabo una persona, de acuerdo a una cultura y tiempo específico. La Educación hoy en día afronta varios problemas, estos han sido atribuidos a los modelos de Enseñanza, al parecer, se mantiene un modelo basado en el positivismo y el occidentalismo, esto debido a que se pensaba que dichos modelos van a mantener la misma eficiencia de años atrás y poder afrontar esta época tan cambiante (ESTRADA GARCIA, 2018b).

A medida que el tiempo pasa, aparecen nuevos problemas que obligan a repensar la forma de enseñar, esto plantea la necesidad de enseñar desde un enfoque inter o transdisciplinar, que permita comprender un problema desde diferentes perspectivas, para dar solución, y que se mantenga en vigencia por mucho tiempo, mas no, solo para ese momento; un ejemplo claro, es la contaminación ambiental, en donde no se ha encontrado un método asertivo para combatir esta problemática, he ahí la falencia del modelo educativo con el que se está enseñando, se mantiene el reduccionismo, por lo que no da paso a crear un conocimiento transdisciplinar, con una diversidad de métodos.

Apegado al análisis, Morin (1999) enfatiza que la complejidad no es, de ningún modo, una respuesta a los problemas epistemológicos de la filosofía; al contrario, es el reconocimiento de las incertidumbres y contradicciones, es un paradigma que desafía al pensamiento, y pone de manifiesto la existencia de una forma de pensar, que permite mirar la realidad desde un enfoque integrado y sistémico. Además, Morin (2007, p. 44) considera que:

[...] la mayor aportación del conocimiento de la centuria pasada ha sido el conocimiento de los límites del conocimiento. La mayor certidumbre que nos ha dado es la de la imposibilidad de eliminar ciertas incertidumbres, no sólo en la acción sino también en el conocimiento. 
En relación a lo citado, se considera que la existencia de incertidumbre dentro del conocimiento es una posibilidad para aprender algo nuevo; la certidumbre hace que el ser humano se conforme con lo aprendido, llevándole a creer que el conocimiento aprendido es incuestionable y lleno de certeza (ESTRADA GARCIA, 2019). Esto, traducido al campo educativo, limita tanto al docente, como al estudiante, a emancipar el conocimiento, a no salir de la zona de confort, por lo que la búsqueda de una nueva vía para generar conocimientos, solo queda en intenciones.

Ante las emergencias de la época no solo en el campo de la Educación, los diferentes paradigmas, enfoques y teorías han planteado un debate en torno a la concepción de la ciencia; algunos aportes, como los de Kosik (1967, p. 13) resaltan que "el acceso al conocimiento científico o filosófico no es considerado de una manera gradual, es decir, implica un salto cualitativo". Unos años más tarde, Bachelard (2000) menciona que "es necesaria una ruptura epistemológica contra el sentido común", lo que conlleva también a una ruptura dentro del conocimiento común, ordinario y científico.

En torno a lo mencionado, se considera que una de las rupturas más importantes ha sido el debilitamiento de la lógica clásica, lo que implica no lograr una descripción de lo lógico-racional de lo real. Esto ha dado apertura a la incorporación de un lenguaje científico más natural, y una investigación menos formal, permitiendo la inserción de un método más flexible, adaptativo y humano.

En el contexto educativo, se debe reestructurar el modelo pedagógico, para que su finalidad sea fortalecer la autonomía, la independencia, el emprendimiento, el liderazgo y la capacidad del estudiante de generar su propio conocimiento; en relación a lo último, Estrada García y Gonzáles Anguieta (2017, p. 240) sugiere que el estudiante está llamado a "[...] autoformarse, aprender a aprender, a través de una metodología interdisciplinaria, utilizando las tecnologías de información y la comunicación". En cuanto a los docentes, Collado Ruano, Madroñero Morillo y Álvarez González (2018) consideran que existen diferentes dimensiones formativas que los docentes del siglo XXI deberían desarrollar para transformar su realidad socioecológica. Además, el autor considera que una Educación transdisciplinar revela la intencionalidad de transgredir el abordaje disciplinar, reconociendo la multidimensionalidad y el dinamismo intrínseco de los fenómenos que interactúan en la formación humana.

Como complemento a lo expuesto, se debe salir de la imposibilidad, pensar en ir más allá de la reducción o disyunción y pensar en conjunto, con la finalidad 
de religar lo fragmentado desde una perspectiva transdisciplinar. Es necesario tener claro que la complejidad no trata de buscar el conocimiento general, más bien, brinda un método para detectar las conexiones, articulaciones existentes en una relación de sistema, este puede ser, la humanidad, la naturaleza, la sociedad o la cultura.

\section{A modo de conclusión}

Las reflexiones preliminares que se ofrecen en este documento se sustentan en la necesidad de repensar la Educación, específicamente el proceso de Enseñanza y aprendizaje, puesto que el modelo de Educación con el que se está enseñando muestra limitaciones, por lo que el estudiante tiene complicaciones para poder adaptarse; cada vez se le hace más difícil alcanzar sus objetivos, puesto que este modelo es unidireccional, considera un solo estilo de aprendizaje para todos los estudiantes, y sobre todo conduce a una especialización en ciertas áreas de conocimiento, dando lugar a la fragmentación de saberes.

Se ve la necesidad de educar mediante un modelo flexible, de diálogo, que permita al estudiante ser el autor de su conocimiento, $\mathrm{y}$ al docente ser el guía del proceso de Enseñanza, en el que el objetivo de la Educación vaya más allá de la simple acumulación de contenidos y cuantificación de calificaciones. Para plasmar estas ideas dentro de la Educación, se toma como fundamento el paradigma del pensamiento complejo, puesto que abre las puertas al análisis y a la reflexión sobre la realidad de nuestro entorno, para a partir de ahí crear nuevos constructos teóricos. También, invita a la sociedad a concientizarse sobre sus acciones, pues es necesario ya que en la época actual, cada vez más se pierden valores, ética y, en general, el sentimiento de solidaridad o compasión por otros seres vivos; se considera que el cambio acelerado de este mundo ha dado origen a seres humanos más individualistas y egoístas.

Para religar lo que está fragmentado y mostrar lo que ha ocultado el paradigma cartesiano positivista (saberes no científicos), es necesario repensar la Educación desde la filosofía y la cosmovisión, con la finalidad de pensar desde las mentes creativas de los estudiantes, enfocados en la propia cosmología de los pueblos y nacionalidades a los que pertenecen, sustentando este proceso en la epistemología de la sabiduría, conocimiento, ciencia y tecnología.

Las instituciones de Educación Superior están llamadas a formar profesionales capaces de desenvolverse en una sociedad interactiva, solucionando los problemas sociales existentes, generando cambios productivos y promoviendo la generación de conocimiento. Para lograr todos estos objetivos y formar futuros profesionales 
capaces de generar un verdadero cambio en la sociedad, debemos modificar el proceso de Enseñanza, hacerlo un proceso sistémico que se enfoque en la transmisión de la cultura dentro de la institución educativa.

El constante desafío es buscar las formas de llevar a la realidad educativa los principios de la complejidad, y poder innovar el proceso de Enseñanza y aprendizaje, con la finalidad de enseñar al estudiante a cuestionar la 'verdad', puesto que, según Morin (2007) ninguna verdad es absoluta, no existe la única y última verdad, por lo que es necesario saber vivir con la incertidumbre, con la posibilidad de que todo es posible según su contexto y la visión del fenómeno.

En general, la Educación está llamada a comprender la Enseñanza de las incertidumbres que han aparecido producto de la disyunción, reducción y especialización (Morin, 2007). De ahí la necesidad de enseñar principios de estrategia que afronten los riesgos, lo inesperado, lo incierto; pues es necesario aprender a navegar en un océano de incertidumbres a través de archipiélagos de certeza y preparar nuestras mentes para esperar lo inesperado y poder afrontarlo. 


\section{Os princípios da complexidade e sua contribuição ao processo de ensino}

\section{Resumo}

O objetivo deste ensaio é propor uma ecologia do conhecimento entre os princípios da complexidade e os paradigmas considerados tradicionais. Em um primeiro momento, é oferecida uma perspectiva do que se entende por complexidade, seguida de uma breve abordagem do processo de ensino e, finalmente, uma sistematização de conceitos entre os princípios da complexidade e o processo de ensino. A hermenêtica foi utilizada como método de pesquisa. Concluindo, o documento integra um diálogo epistemológico que orienta repensar a Educação, especificamente o processo de ensino, a partir de uma perspectiva complexa e transdisciplinar.

Palavras-chave: Ensino Superior. Pensamento Complexo. Processo de Ensino. Princípios de Pensamento Complexo.

\section{The principles of complexity and their contribution to the Teaching process}

\section{Abstract}

The objective of this essay is to propose an ecology of knowledge between the principles of complexity and the paradigms considered as traditional. In the first instance, a perspective of what is understood by complexity is offered, followed by a brief approach to the Teaching process; finally, a systematization of concepts between the principles of complexity and the Teaching process is carried out. Hermeneutics was used as a research method. In conclusion, the document integrates an epistemological dialogue that guides rethinking Education, specifically the Teaching process, from a complex and transdisciplinary perspective.

Keywords: Higher Education. Complex Thinking. Teaching Process. Principles of Complex Thinking. 


\section{Referencias}

ASAMBLEA NACIONAL DEL ECUADOR. Constitución Política del Ecuador 2008. Montecristi: Secretaria General de la Asamblea Nacional, 2008 .

ASAMBLEA NACIONAL DEL ECUADOR. Ley Orgánica de Educación Superior. Quito: Secretaria General de la Asamblea Nacional, 2010.

BACHELARD, G. La formación del espíritu cientifico. Buenos Aires: Argos, 2000.

BISQUERRA ALZINA, R. Orientación, tutoría y educación emocional. Madrid: Síntesis, 2012.

BOHÓRQUEZ ARENAS, L. A.; D’AMORE, B. Factores que apoyan o limitan los cambios de concepciones de los estudiantes para profesor de matemática sobre la gestión del proceso de Enseñanza-aprendizaje. Avances de Investigación en Educación Matemática, [s. 1.], n. 13, p. 85-103, mayo 2018. https://doi.org/10.35763/aiem.v0i13.228

BORRERO SPRINGER, R. Y; GAMBOA GRAUS, M. E. Influencia de los organizadores del curriculum en la planificación de la contextualización didáctica de la matemática. Boletín Redipe, [s. 1.], v. 6, n. 1, p. 90-112, 30 Ene. 2017. Disponible en: https://goo.gl/bh3Qwz. Acceso en: 19 mayo 2018.

CEDEÑO ÁlVAREZ, R. C.; GUARNIZO DELGADO, J. B.; PACHECO PROAÑO, I. S. Adopción del recurso teatral en el tratamiento didáctico del proceso de Enseñanza aprendizaje devenido experiencia axiológica. Revista Científica Mundo de la Investigación y el Conocimiento, [s. 1.], v. 2, n. 2, p. 761-781. Disponible en: https://goo.gl/5i14hK. Acceso en: 7 mayo 2018.

COLLADO RUANO, J.; MADROÑERO MORILLO, M.; ÁLVAREZ GONZALEZ, F. Educación transdisciplinar: formando en competencias para el buen vivir. Ensaio: Avaliação e Políticas Públicas em Educação, Rio de Janeiro, v. 26, n. 100, p. 619-644, jul.-set. 2018. https://doi.org/10.1590/s0104-40362018002601487

CONSEJO DE EDUCACIÓN SUPERIOR. Construcción del nuevo régimen académico: Gestión Curricular. Quito: Secretaria Nacional de Educación Superior, Ciencia y Tecnología e Innovación, 2015. 
ESTRADA GARCIA, A. Educación superior: una perspectiva desde el pensamiento complejo. Saarbrücken: Académica Española, 2019.

ESTRADA GARCIA, A. Estilos de aprendizaje y rendimiento académico. Revista Boletín Redipe, [s. 1.], v. 7, n.7, p. 218-228, jul. 2018b. Disponoble en: https://goo.gl/D3ZA8E. Acceso en: 25 feb. 2018.

ESTRADA GARCIA, A. Pensamiento complejo y desarrollo de competencias transdisciplinares en la formación profesional. Revista Arbitrada del Centro de Investigación y Estudios Gerenciales, [s. 1.], v, 5, n. 31, p. 98-108, enero-marzo 2018a. Disponoble en: https://goo.gl/nNSY1C. Acceso en: 24 abr. 2018.

ESTRADA GARCIA, A.; GONZÁLES ANGUIETA, A. Respuesta a la matriz productiva desde la educación en ciencia, tecnología y sociedad (ECTS) y la metodología sistémica. Revista Arbitrada del Centro de Investigación y Estudios Gerenciales, [s. 1.], v. 5, n. 27, p. 236-249., sept. 2017. Disponible en: https://goo.gl/aid1Qd. Acceso en: 24 feb. 2018.

GÓMEZ, C.; HERNÁNDEZ, M.; RAMOS, R. Principios epistemológicos para la Enseñanza aprendizaje, según el pensamiento complejo de Edgar Morin. Pueblo Continente, [s. 1.], p. 471-479, 2016. Disponible en: http://journal.upao.edu.pe/PuebloContinente/article/view/699. Acceso en: 23 feb. 2018.

GONFIANTIN, V. Prólogo. In: Yanes Guzmán, J. Complejidad y calidad de la educación. Santiago de Chile: RIL, 2015. p. 5-8.

GUZMÁN, F. Estudio sobre los usos didácticos procesos informáticos. Barcelona: Inde, 2011.

HIMMEL, E. La evaluación de aprendizajes en la educación superior. Revista Calidad en la Educación, [s. 1.], n. 15, p. 1-8, 2001. https://doi.org/10.31619/caledu.n15.447

KOSIK, K. Dialéctica de lo concreto. México: Grijalbo, 1967.

MAASEN, S; LIEVEN, O. Transdisciplinarity: a new way of governing science? Science and Public Policies, Oxford, v. 33, n. 6, p. 399-410, July 2016. https://doi.org/10.3152/147154306781778803

MORA, F. Neuroeducación: solo se puede aprender aquello que se ama. Madrid: Alianza, 2019. 
MORIN, E. ¿Cómo vivir en tiempos de crisis? Buenos Aires: Nueva Visión, 2011.

MORIN, E. Introducción al pensamiento complejo. Barcelona: Gedisa, 1994.

MORIN, E. Introducción al pensamiento complejo. Buenos Aires:

Gedisa, 1995.

MORIN, E. La cabeza bien puesta: repensar la reforma. Buenos Aires: Nueva Visión, 2007.

MORIN, E. Los siete saberes necesarios para la educación del futuro. París: Unesco, 1999.

OSORIO GARCÍA, S. N. El pensamiento complejo y la transdisciplinariedad: fenómenos emergentes de una nueva racionalidad. Revista de la Facultad de Ciencias Económicas: Investigación y Reflexión, Bogotá, v. 20, n. 1, p. 269-291, enero-jun. 2012. Disponible en: https://goo.gl/qqQMMs. Acceso en: 23 feb. 2018.

PERAZA ZAMORA, C. et al. Caracterización de los medios de Enseñanza en el proceso de Enseñanza- aprendizaje en la Educación Física. Revista de Ciencia y Tecnología, v. 12, n. 1, p. 4-11, ago. 2017. Disponible en: https://goo.gl/6Y5H7K. Acesso en: 2 jun. 2018.

SERGHIE, D. Context in collaborative structures: transdisciplinarity. Network Intelligence Studies, [s. 1.], v. 1, n. 2, p. 5-12, oct. 2015. Disponible em: http://seaopenresearch.eu/Journals/articles/NIS_2_1.pdf. Acceso en: 16 jun 2018.

TEJEDOR TEJEDOR, F. La evaluación del profesorado como estrategia de mejora de la calidad de la Enseñanza universitaria. Revista de Educación y Derecho, Barcelona, n. 17, p. 1-19, feb. 2018. Disponible en: https://goo.gl/eoKdhv. Acceso en: 4 mayo 2018.

TORREALBA, C.; PÉREZ, P.; CASTILLO, N. El pensamiento complejo y la transcomplejidad: visión emergente en el desarrollo de un enfoque epistemológico en la investigación educativa. Revista Arbitrada del Centro de Investigación y Estudios Gerenciales, n. 33, p. 139-154, sept. 2018. Disponible en: https://goo.gl/iHrbo2. Acceso en: 18 jun. 2018.

UNESCO. Las tecnologías de la información y la comunicación en la formación docente: guía de planificación. Paris, 2004. 
UNESCO. Rethinking education: towards a global common good? Paris, 2015.

VANOLI, F. El pensamiento complejo y la transdisciplina en el abordaje del hábitat. Reflexiones en torno a la experiencia del Seminario de Gestión Territorial y Hábitat. Revista Pensum, [s. 1.], v. 3, n. 3, p. 141-146, 2017. Disponible en: https://revistas.unc.edu.ar/index.php/pensu/article/view/19033. Acceso en: 4 jun. 2018.

YANES, J. Complejidad y calidad de la educación. Santiago de Chile: Ril, 2015.

ZABALZA, M. El proceso de Enseñanza aprendizaje: el acto didáctico. Cataluña Universidad Rovira I Virgili, 2001.

\section{Información del autor}

Alex Estrada García: Doctorando en Educación por el Instituto de Estudios Universitarios, en México. Maestro en Transdisciplinariedad y Complejidad del Conocimiento, Multiversidad Mundo Real Edgar Morin, en México. Contacto: alexdario94@hotmail.es (iD http://orcid.org/0000-0001-5278-8221 